\title{
A RELATION BETWEEN INVARIANT MEANS ON LIE GROUPS AND INVARIANT MEANS ON THEIR DISCRETE SUBGROUPS ${ }^{1}$ \\ BY
}

JOHN R. GROSVENOR

\begin{abstract}
Let $G$ be a Lie group, and let $D$ be a discrete subgroup of $G$ such that the right coset space $D \backslash G$ has finite right-invariant volume. We will exhibit an injection of left-invariant means on $l^{\infty}(D)$ into left-invariant means on the left uniformly continuous bounded functions of $G$. When $G$ is an abelian Lie group with finitely many connected components, we also show surjectivity, and when $G$ is the additive group $\mathbf{R}^{n}$ and $D$ is $\mathbf{Z}^{n}$, the bijection will explicitly take the form of an integral over the unit cube $[0,1]^{n}$.
\end{abstract}

1. Introduction. This paper grew out of an attempt at the still unsolved problem of parametrizing invariant means on $l^{\infty}(\mathbf{Z})$, where $\mathbf{Z}$ is the discrete additive group of integers. This led to the more general problem of relating left-invariant means on $l^{\infty}(D)$ and left-invariant means on the left uniformly continuous bounded functions of $G\left(\mathrm{UCB}_{1}(G)\right)$, where $G$ is a Lie group and $D$ a (not necessarily normal) discrete (hence closed) subgroup, where the coset space $G / D$ (or $D \backslash G$ ) is compact. In fact, in $\S 3$ it will be shown that if $G$ is a locally compact, second countable topological group (in particular, a Lie group), and the right coset space $D \backslash G$ has right-invariant finite volume, we can construct an injection of left-invariant means (LIM's) on $l^{\infty}(D)$ into LIM's on $\mathrm{UCB}_{1}(G)$. In $\S 4$ we consider the case where $G=\mathbf{R}^{n}$ and $D=\mathbf{Z}^{n}$ and prove that the injection of $\S 3$ from invariant means on $l^{\infty}(\mathbf{Z})$ to invariant means on the uniformly continuous bounded functions of $\mathbf{R}^{n}\left(\operatorname{UCB}\left(\mathbf{R}^{n}\right)\right)$ is also surjective, showing that every invariant mean on $\operatorname{UCB}\left(\mathbf{R}^{n}\right)$ can be constructed from one on $l^{\infty}\left(\mathbf{Z}^{n}\right)$ and conversely. Thus, if the parametrization problem for $l^{\infty}(\mathbf{Z})$ is solved, the analogous problem for $\mathrm{UCB}(\mathbf{R})$ will also be solved.

Using the result of $\S 4$, we show, in $\S 5$, that there is a bijection between invariant means on $l^{\infty}(D)$ and invariant means on $\operatorname{UCB}(G)$ when $G$ is an abelian Lie group with finitely many connected components. Finally, in $\$ 6$ we examine the problem of generalizing the surjectivity proof for $\mathbf{R}^{n}$ to the general Lie group.

I am deeply indebted to my dissertation advisor, Professor Leonard L. Scott, Jr., for the many hours he devoted to me in this undertaking and to the referee for arguments used in the proofs of Lemma 3.1 and Theorem 3.2.

Received by the editors July 6, 1983 and, in revised form, June 25, 1984.

1980 Mathematics Subject Classification. Primary 43A07; Secondary 22E15, 22E40.

Key words and phrases. Invariant mean, topological invariant mean.

${ }^{1}$ This paper is based on a Ph.D. dissertation written by the author at the University of Virginia under the supervision of Professor Leonard L. Scott, Jr. 
2. Preliminaries and notation. Let $G$ be a locally compact topological group with fixed left Haar measure. The vector space of equivalence classes of essentially bounded real-valued functions on $G$ is denoted by $\mathbf{L}^{\infty}(G)$, and the set of real-valued functions $\phi$ on $G$ for which $\int_{G}|\phi(g)| d g<\infty$ is denoted $L^{1}(G)$. For $\phi \in L^{1}(G)$, define $\tilde{\phi}(x)=\phi\left(x^{-1}\right)$. It is easy to see that if $f: G \rightarrow \mathbf{R}$ is essentially bounded, so are the convolutions $\phi * f$ and $f * \tilde{\phi}$ defined by

$$
(\phi * f)(s)=\int_{G} f\left(t^{-1} s\right) \phi(t) d t
$$

and

$$
(f * \tilde{\phi})(s)=\int_{G} f(t) \tilde{\phi}\left(t^{-1} s\right) d t=\int_{G} f(t) \phi\left(s^{-1} t\right) d t
$$

Define $P(G)$ to be $\left\{\phi \in L^{1}(G): \phi \geqslant 0\right.$ and $\left.\int_{G} \phi(t) d t=1\right\}$.

The left and right actions of $G$ on $\mathbf{L}^{\infty}(G)$ are denoted by, respectively, $x \cdot f(y)=$ $f\left(x^{-1} y\right)$ and $f \cdot x(y)=f\left(y x^{-1}\right)$ for $x, y \in G$ and $f \in \mathbf{L}^{\infty}(G)$. A function $f \in \mathbf{L}^{\infty}(G)$ is left [resp., right] uniformly continuous if, given $\varepsilon>0$, there is a neighborhood $U(\varepsilon)$ of the identity element of $G$ such that $\|x \cdot f-f\|_{\infty}<\varepsilon\left[\right.$ resp., $\left.\|f \cdot x-f\|_{\infty}<\varepsilon\right]$ for all $x \in U(\varepsilon)$. (Note that Greenleaf's definitions [5, p. 21] are the reverse of the above.) The left and right uniformly continuous bounded functions on $G$ are denoted by, respectively, $\mathrm{UCB}_{1}(G)$ and $\operatorname{UCB}_{\mathrm{r}}(G)$. The set of uniformly continuous bounded functions on $G, \mathrm{UCB}(G)$, is defined to be $\mathrm{UCB}_{1}(G) \cap \mathrm{UCB}_{\mathrm{r}}(G)$.

If $W(G)$ is a closed subspace of $\mathbf{L}^{\infty}(G)$ containing the constant function $e(x) \equiv 1$, an element $m$ of $W(G)^{*}$ is called a mean if $m$ is positive and $m(e)=1$. It is a left-invariant mean (LIM) [resp., right-invariant mean (RIM)] if, given $f \in W(G)$, $m(x \cdot f)=m(f)$ [resp., $m(f \cdot x)=m(f)$ ] for all $x \in G$, and it is a topological left-invariant mean (TLIM) [resp., topological right-invariant mean (TRIM)] if, given $f \in W(G), m(\phi * f)=m(f)$ [resp., $m(f * \tilde{\phi})=m(f)]$ for all $\phi \in P(G)$. It is well known that if there is a LIM on one of $\mathrm{L}^{\infty}(G), \mathrm{CB}(G)=$ continuous bounded functions of $G, \mathrm{UCB}_{1}(G)$, or $\operatorname{UCB}(G)$, there is a $\operatorname{LIM}$ on any of the others [8, p. 26]. In such a case we say $G$ is amenable.

The following well-known results [5, pp. 24, 27] are stated here for later quotations:

LEMmA 2.1. If $f \in \mathbf{L}^{\infty}(G)$ and $\phi \in P(G)$, then $\phi * f \in \operatorname{UCB}(G)$ and $f * \tilde{\phi} \in$ $\mathrm{UCB}_{\mathrm{r}}(G)$. If $g \in \mathrm{UCB}_{\mathrm{r}}(G)$ [resp., $g \in \mathrm{UCB}_{1}(G)$ ], then $\phi * g[$ resp., $g * \tilde{\phi}]$ is in $\mathrm{UCB}(G)$.

LEMMA 2.2. If $m$ is a LIM on $\operatorname{UCB}_{1}(G)$, then it is also a TLIM on $\operatorname{UCB}_{1}(G)$. The same result is true for $\mathrm{UCB}(G)$ or for $m$ any left-invariant continuous linear functional on $\mathrm{UCB}_{1}(G)$ or $\operatorname{UCB}(G)$.

Because $\operatorname{dim} \mathrm{UCB}_{1}(G)$ is infinite, the kernel of each of these invariant means has infinite dimension. The following corollary actually describes the functions contained in the intersection of all these kernels. 
THEOREM 2.3. Let $N_{G}$ be the set of all left-invariant means on $\operatorname{UCB}_{1}(G)$. Then $\bigcap_{m \in N_{G}}$ ker $m$ is the closure $\overline{\langle f-x \cdot f\rangle}$ of the space spanned by the differences $f-x \cdot f$, with $f \in \mathrm{UCB}_{1}(G)$ and $x \in G$.

Proof. Let $l$ be a left-invariant continuous linear functional on $\operatorname{UCB}_{1}(G)$. Extend $l$ to a left-invariant continuous linear functional $l_{e}$ on $\mathbf{L}^{\infty}(G)$ by letting $l_{e}(f)=$ $l(\phi * f)$, where $\phi \in P(G)$ is fixed. By Theorem IV 16 of [4], $l_{e}(f)=\int_{G} f d \nu$, where $\nu$ is a bounded, finitely additive set function. Since $l_{e}(f)=l_{e}(x \cdot f)$ for each $f$ and all $x \in G$,

$$
\int_{G} f(y) d \nu(y)=\int_{G}(x \cdot f)(y) d \nu(y)=\int_{G} f\left(x^{-1} y\right) d \nu(y)=\int_{G} f(z) d \nu(x z)
$$

in particular, for $f=\chi_{E}$, the characteristic function of the set $E$, we get $\nu(E)=$ $\nu(x E)$. By the Jordon decomposition Theorem III 8 of [4], $\nu=\nu^{+}-\nu^{-}$, where $\nu^{+}, \nu^{-}$ are positive, $\nu^{+}(E)=\sup _{F \subset E} \nu(F)$, and $\nu^{-}(E)=-\inf _{F \subset E} \nu(F)$ so that

$$
\nu^{+}(x E)=\sup _{F \subset x E} \nu(F)=\sup _{x^{-1} F \subset E} \nu(F)=\sup _{x^{-1} F \subset E} \nu\left(x^{-1} F\right)=\nu^{+}(E) .
$$

Similarly, $\nu^{-}(x E)=\nu^{-}(E)$; thus,

$$
l_{e}(f)=\int_{G} f d \nu^{+}-\int_{G} f d \nu^{-}=l_{e}^{+}(f)-l_{e}^{-}(f),
$$

with $l_{e}^{+}, l_{e}^{-}$positive and left-invariant, so they are scalar multiples of LIM's.

Now let

Suppose $l \in \operatorname{UCB}(G)^{*}$ vanishes on

$$
g \in \bigcap_{m \in N_{G}} \operatorname{ker} m
$$

$$
\overline{\langle f-x \cdot f\rangle}_{f \in \mathrm{UCB}_{l}(G)}
$$

then $l(g)=l(x \cdot g)$ and, by Lemma 2.2 and the above,

$$
l(g)=l(\phi * g)=l_{e}(g)=l_{e}^{+}(g)-l_{e}^{-}(g),
$$

and since $l_{e}^{+}, l_{e}^{-}$are scalar multiples of means, $l_{e}^{+}(g)=0=l_{e}^{-}(g)$, so $l(g)=0$. By a corollary to the Hahn-Banach theorem, $g \in \overline{\langle f-x \cdot f\rangle}$. Since each ker $m$ is closed, it is clear that $\overline{\langle f-x \cdot f\rangle} \subset \bigcap_{m \in N_{G}}$ ker $m$.

THEOREM 2.4. Let $W$ be the subspace of those functions in $\mathrm{UCB}(\mathbf{R})$ whose antiderivatives belong to $\mathrm{UCB}(\mathbf{R})$. Then $W \subset \bigcap_{m \in N_{\mathbf{R}}} \operatorname{ker} m$.

Proof. Let $m$ be any LIM on $\operatorname{UCB}(\mathbf{R})$, so by Lemma $2.2, m$ is also a TLIM. Let $f \in W$, and let $\chi_{[0,1]}$ be the characteristic function of the interval [0,1]. Then $\left(\chi_{[0,1]} * f\right)(s)=\int_{0}^{1} f(s-t) d t$. Let $s-t=r$ and get

$$
\int_{s-1}^{s} f(r) d r=F(s)-F(s-1)=(F-1 \cdot F)(s)
$$

where $F^{\prime}=f$, so

$$
m(f)=m\left(\chi_{[0,1]} * f\right)=m(F-1 \cdot F)=m(F)-m(1 \cdot F)=0
$$

by left invariance of $m$. Since $m$ was arbitrary, $f \in \bigcap_{m \in N_{\mathbf{R}}}$ ker $m$.

Theorems 2.3 and 2.4 are due to the author. Combining the results of these two theorems, we see that a uniformly continuous bounded function on $\mathbf{R}$ having a 
bounded antiderivative is a uniform limit of functions of the form $\sum_{i=1}^{k} a_{i}\left(f_{i}-x_{i} \cdot f_{i}\right)$, where each $f_{i} \in \mathrm{UCB}(\mathbf{R})$. For example,

$$
f(x)=\sin x=\frac{1}{2}(\sin x-(-\pi / 2) \cdot \sin x)+\frac{1}{2}(\cos x-(-\pi / 2) \cdot \cos x) .
$$

3. Injecting LIM's on $l^{\infty}(D)$ into LIM's on $\mathrm{UCB}_{1}(G)$. Let $G$ be a locally compact, second countable (hence $\sigma$-compact), amerable topological group and $D$ a discrete (hence countable) subgroup of $G$. Then there is a Borel measurable transversal $K$ (which can be taken to be $\sigma$-compact) for the right coset space $D \backslash G$ with cross-sectional transformation $\tau: D \backslash G \rightarrow K$. By definition, $D K=G$. $K$ can be taken so that $\left(\overline{K^{0}}\right) \supset K$, where $K^{0}$ denotes the interior of $K$.

If $D \backslash G$ has finite right-invariant volume $\nu$ (which will be taken to be normalized), let $\nu_{0}$ be the measure on $K$ preserved by $\tau$. Let $C_{c}(G)$ denote the continuous real-valued functions on $G$ with compact support. Let $h \in C_{c}(G) \subset \mathrm{UCB}(G)$ be a symmetric function for which $h \geqslant 0, \operatorname{supp} h \subset K^{0}$, and $\int_{K} h(x) d \nu_{0}(x)=1$.

Lemma 3.1. Given $f \in l^{\infty}(D)$, the function $T f$ defined by

is in $\mathrm{UCB}_{1}(G)$.

$$
T f(g)=\sum_{d_{0} \in D} f\left(d_{0}\right) h\left(d_{0} g^{-1}\right)
$$

Proof. Let $U$ be a symmetric neighborhood of the unit in $G$ such that $(\overline{U \cdot \operatorname{supp} h})$ is contained in $K^{0}$. If $x \in U$ and $g \in G$, then

$$
\begin{aligned}
|T f(x g)-T f(g)| & =\left|\sum_{d_{0} \in D} f\left(d_{0}\right) h\left(d_{0}(x g)^{-1}\right)-\sum_{d_{0} \in D} f\left(d_{0}\right) h\left(d_{0} g^{-1}\right)\right| \\
& =\left|\sum_{d_{0} \in D} f\left(d_{0}\right)\left(h\left(d_{0} g^{-1} x^{-1}\right)-h\left(d_{0} g^{-1}\right)\right)\right|
\end{aligned}
$$

however,

$$
h\left(d_{0} g^{-1} x^{-1}\right) \neq 0 \Leftrightarrow d_{0} g^{-1} x^{-1} \in \operatorname{supp} h \Leftrightarrow g^{-1} \in d_{0}^{-1} \operatorname{supp} h \cdot U \subset d_{0}^{-1} K,
$$

possible for only one $d_{0}^{*} \in D$. All terms except for one vanish, and the sum is thus

$$
\left|f\left(d_{0}^{*}\right)\left(h \cdot x\left(d_{0}^{*} g^{-1}\right)-h\left(d_{0}^{*} g^{-1} j\right)\right)\right| \leqslant\|f\|_{\infty}\|h \cdot x-h\|_{\infty} .
$$

By further restricting the size of $U$ this can be made as small as desired.

THEOREM 3.2. Let $m$ be a LIM on $l^{\infty}(D)$, where $D \backslash G$ has right-invariant finite normalized volume $\nu$. If $m_{e}(f)=\int_{K} m\left((t \cdot f)_{\mathrm{r}}\right) d \nu_{0}(t)$ for $f \in \mathrm{UCB}_{1}(G)$, then $m \rightarrow$ $m_{e}$ is an injection of LIM's on $l^{\infty}(D)$ into LIM's on $\mathrm{UCB}_{1}(G)$.

Let $\phi(D t)=m\left((t \cdot f)_{\mathrm{r}}\right)=\phi_{0}(t)$. Then $\phi$ is well defined, because if $D t_{1}=D t_{2}$ we have

$$
\begin{aligned}
t_{2} t_{1}^{-1} \in D & \Rightarrow \phi\left(d t_{1}\right)=m\left(\left(t_{1} \cdot f\right)_{\mathrm{r}}\right)=m\left(t_{2} t_{1}^{-1} \cdot\left(t_{1} \cdot f\right)_{\mathrm{r}}\right) \quad \text { by left invariance } \\
& =m\left(\left(t_{2} t_{1}^{-1} t_{1} \cdot f\right)_{\mathrm{r}}\right)=m\left(t_{2} \cdot f\right)=\phi(D t),
\end{aligned}
$$

so

$$
\int_{K} m\left((t \cdot f)_{\mathrm{r}}\right) d \nu_{0}(t)=\int_{K} \phi_{0}(t) d \nu_{0}(t)=\int_{D \backslash G} \phi(D t) d \nu(D t),
$$


so $m_{e}$ is a mean and left-invariant because, given $f \in \mathrm{UCB}_{1}(G)$, for any $x \in G$ we have

$$
\begin{aligned}
m_{e}(x \cdot f) & =\int_{K} m\left((t \cdot(x \cdot f))_{\mathrm{r}}\right) d \nu_{0}(t)=\int_{K} m\left((t x \cdot f)_{\mathrm{r}}\right) d \nu_{0}(t) \\
= & \left.\int_{D \backslash G} \phi(D t x) d \nu(D t)=\int_{D \backslash G} \phi(D t x) d \nu(D t x) \quad \text { (by right invariance of } \nu\right) \\
= & \int_{D \backslash G} \phi(D s) d \nu(D s) \quad(s=t x) \\
= & \int_{K} m\left((s \cdot f)_{\mathrm{r}}\right) d \nu_{0}(s)=m_{e}(f) .
\end{aligned}
$$

Now, if $f \in \operatorname{UCB}_{1}(G)$ consider the function $T f$ of Lemma 3.1. Note that by the support condition on $h$, if $t \in K$ and $d_{0} \in D$, then $h\left(d_{0} t\right) \neq 0 \Rightarrow d_{0} t \in K \Rightarrow d_{0}$ is the identity by the definition of $K$. Thus,

$$
(t \cdot T f)_{\mathrm{r}}(d)=T f\left(t^{-1} d\right)=\sum_{d_{0} \in D} f\left(d_{0}\right) h\left(d_{0} d^{-1} t\right) .
$$

Letting $d_{0} d^{-1}=c_{0}$, we have $d_{0}=c_{0} d$, and as $d_{0}$ ranges over $D$, so does $c_{0}$, giving us

$$
\sum_{c_{0} \in D} f\left(c_{0} d\right) h\left(c_{0} t\right)=f(d) h(t)=(h(t) f)(d)
$$

since all terms vanish except for $c_{0} t \in K$; i.e., $c_{0}=$ the identity. This gives

$$
m\left((t \cdot T f)_{\mathrm{r}}\right)=m(h(t) f)=h(t) m(f)
$$

so

$$
\begin{aligned}
m_{e}(T f) & =\int_{K} m\left((t \cdot T f)_{\mathrm{r}}\right) d \nu_{0}(t)=\int_{K} m(f) h(t) d \nu_{0}(t) \\
& =m(f) \int_{K} h(t) d \nu_{0}(t)=m(f) .
\end{aligned}
$$

It thus follows immediately that $m \rightarrow m_{e}$ is injective. Q.E.D.

4. LIM's on $l^{\infty}\left(\mathbf{Z}^{n}\right)$ and on $\operatorname{UCB}\left(\mathbf{R}^{n}\right)$. The first step in constructing a bijection between LIM's on $l^{\infty}(D)$ and on $\operatorname{UCB}(G)$ when $G$ is a connected abelian Lie group and $D$ a discrete subgroup for which $G / D(=D \backslash G)$ has right-invariant finite volume (hence is compact, since $G / D$ is a group) is to prove that the relation $m \rightarrow m_{e}$ of the previous section is bijective when $G=\mathbf{R}^{n}$ and $D=\mathbf{Z}^{n}$.

THEOREM 4.1. Let $m$ be a LIM on $l^{\infty}\left(\mathbf{Z}^{n}\right)$ and, for $f \in \mathrm{UCB}(\mathbf{R})$, define

$$
m_{e}(f)=\int_{[0,1]^{n}} m\left(\left(\left(t_{1}, \ldots, t_{n}\right) \cdot f\right)_{\mathrm{r}}\right) d t_{1} \cdots d t_{n} .
$$

Then $m \rightarrow m_{e}$ is a bijection between LIM's on $l^{\infty}\left(\mathbf{Z}^{n}\right)$ and LIM's on $\operatorname{UCB}\left(\mathbf{R}^{n}\right)$.

Proof. We can take $K$ to be $[0,1)^{n}$ in the proof of Theorem 3.2, in which case $\int_{K} m\left((t \cdot f)_{\mathrm{r}}\right) d t$ is the above integral. Thus, $m \rightarrow m_{e}$ is injective.

To show surjectivity, for $j=0, \ldots, n-1$, we prove that $\mu \rightarrow \mu^{(j+1)}$, where

$$
\mu^{(j+1)}(f)=\int_{0}^{1} \mu\left(\left(t_{j+1} \cdot f\right)_{\mathrm{r}}\right) d t_{j+1}
$$


is a surjection of LIM's on $\operatorname{UCB}\left(\mathbf{R}^{j} \oplus \mathbf{Z}^{n-j}\right)$ onto LIM's on $\operatorname{UCB}\left(\mathbf{R}^{j+1} \oplus \mathbf{Z}^{n-j-1}\right)$, where $t_{j+1}$ denotes $t_{j+1} \in \mathbf{R}$ in the $(j+1)$ st position, 0 elsewhere. It is clear that $\mu^{(j+1)}$ is a mean when $\mu$ is. To see that $\mu^{(j+1)}$ is invariant, let

$$
\left(u_{1}, \ldots, u_{j}, u_{j+1} ; k_{1}, \ldots, k_{n-j-1}\right) \in \mathbf{R}^{j+1} \oplus \mathbf{Z}^{n-j-1},
$$

and let $\left(u_{1}, \ldots, u_{j}\right)=U,\left(k_{1}, \ldots, k_{n-j-1}\right)=K$, so that

$$
\begin{aligned}
\mu^{(j+1)}\left(\left(U ; u_{j+1} ; K\right) \cdot f\right) & =\int_{0}^{1} \mu\left(\left(\left(U ; u_{j+1} ; K\right) \cdot t_{j+1} \cdot f\right)_{\mathrm{r}}\right) d t_{j+1} \\
& =\int_{0}^{1} \mu\left(\left(\left(U ; u_{j+1}+t_{j+1} ; K\right) \cdot f\right)_{\mathrm{r}}\right) d t_{j+1} \\
& =\int_{0}^{1}\left(\left(\left(u_{j+1}+t_{j+1}\right) \cdot f\right)_{\mathrm{r}}\right) d t_{j+1}
\end{aligned}
$$

by invariance of $\mu$; letting $u_{j+1}+t_{j+1}=v_{j+1}$, the integral becomes

$$
\int_{u_{j+1}}^{u_{j+1}+1} \mu\left(\left(v_{j+1} \cdot f\right)_{\mathrm{r}}\right) d v_{j+1}=\int_{u_{j+1}}^{0}+\int_{0}^{1}+\int_{1}^{u_{j+1}+1}=\int_{0}^{1}-\int_{0}^{u_{j+1}}+\int_{1}^{u_{j+1}+1} .
$$

Letting $v_{j+1}=s_{j+1}+1$ in the third integral, we get

$$
\int_{0}^{u_{j+1}} \mu\left(1 \cdot\left(s_{j+1} \cdot f\right)_{\mathrm{r}}\right) d s_{j+1}=\int_{0}^{u_{j+1}} \mu\left(\left(s_{j+1} \cdot f\right)_{\mathrm{r}}\right) d s_{j+1}
$$

by left invariance of $\mu$. This integral cancels with the previous integral, so we are left with

$$
\int_{0}^{1} \mu\left(\left(v_{j+1} \cdot f\right)_{\mathrm{r}}\right) d v_{j+1}=\mu_{(j+1)}(f)
$$

thus, $\mu_{(j+1)}$ is left-invariant.

Let $\nu$ be a LIM on $\operatorname{UCB}\left(\mathbf{R}^{j+1} \oplus \mathbf{Z}^{n-j-1}\right)$. If $\mu$ is any $\operatorname{LIM}$ on $\mathbf{L}^{\infty}\left(\mathbf{R}^{j+1} \oplus \mathbf{Z}^{n-j-1}\right)$, define $\mu_{(j+1)}$ on $\mathrm{UCB}\left(\mathbf{R}^{j} \oplus \mathbf{Z}^{n-j}\right)$ by $\mu_{(j+1)}(g)=\mu\left(g_{(j+1)}\right)$, where

$$
g\left(x_{1}, \ldots, x_{j} ; x_{j+1} ; m_{1}, \ldots, m_{n-j}\right)=g\left(x_{1}, \ldots, x_{j} ;\left[x_{j+1}\right] ; m_{1}, \ldots, m_{n-j}\right),
$$

$\left[x_{j+1}\right]$ denoting the greatest integer less than or equal to $x_{j+1}$. Since $k_{j+1} \cdot g_{(j+1)}=$ $\left(k_{j+1} \cdot g\right)_{(j+1)}, \mu_{(j+1)}$ is invariant. Furthermore, $\hat{\nu}$, defined by $\hat{\nu}(h)=\nu\left(\chi_{[0,1]^{j+1}} * h\right)$, gives a TLIM on $\mathbf{L}^{\infty}\left(\mathbf{R}^{j+1} \oplus \mathbf{Z}^{n-j-1}\right)$ (hence, a LIM, by [5, p. 25]). Now, if $f \in \mathrm{UCB}\left(\mathbf{R}^{j+1} \oplus \mathbf{Z}^{n-j-1}\right)$, we have

$$
\begin{aligned}
\left(\hat{\nu}_{(j+1)}\right)^{(j+1)}(f) & =\int_{0}^{1} \hat{\nu}_{(j+1)}\left(\left(t_{j+1} \cdot f\right)_{\mathrm{r}}\right) d t_{j+1} \\
& =\int_{0}^{1} \hat{v}\left(\left(t_{j+1} \cdot f\right)_{\mathrm{r}(j+1)}\right) d t_{j+1} \\
& =\int_{0}^{1} \nu\left(\chi_{[0,1]^{j+1}} *\left(t_{j+1} \cdot f\right)_{\mathrm{r}(j+1)}\right) d t_{j+1} \\
& =\nu\left(\int_{0}^{1}\left(\chi_{[0,1]^{j+1}} *\left(t_{j+1} \cdot f\right)_{\mathrm{r}(j+1)}\right) d t_{j+1}\right),
\end{aligned}
$$


where the last integral is the weak vector-valued integral (see [5, p. 101]). Now,

$$
\begin{aligned}
& \left(\int_{0}^{1} \chi_{[0,1]^{j+1}} *\left(t_{j+1} \cdot f\right)_{\mathrm{r}(j+1)} d t_{j+1}\right)\left(s_{1}, \ldots, s_{j+1} ; m_{1}, \ldots, m_{n-j-1}\right) \\
& \quad=\int_{0}^{1} \chi_{[0,1]^{j+1}} *\left(t_{j+1} \cdot f\right)_{\mathrm{r}(j+1)}\left(s_{1}, \ldots, s_{j+1} ; m_{1}, \ldots, m_{n-j-1}\right) d t_{j+1} \\
& \quad=\int_{0}^{1} \int_{[0,1]^{j+1}}\left(t_{j+1} \cdot f\right)_{\mathrm{r}(j+1)}\left(s_{1}-u_{1}, \ldots, s_{j+1}-u_{j+1}-u_{j+1} ; J\right) d U d t_{j+1} \\
& \quad\left(\text { where } d U=d u_{1} \cdots d u_{j+1}, J=m_{1}, \ldots, m_{n-j-1}\right) \\
& =\int_{0}^{1} \int_{[0,1]^{j+1}}\left(t_{j+1} \cdot f\right)\left(s_{1}-u_{1}, \ldots, s_{j}-u_{j} ;\left[s_{j+1}-u_{j+1}\right] ; J\right) d U d t_{j+1} \\
& =\int_{0}^{1} \int_{[0,1]^{j+1}} f\left(s_{1}-u_{1}, \ldots, s_{j}-u_{j} ;\left[s_{j+1}-u_{j+1}\right]-t_{j+1} ; J\right) d U d t_{j+1} \\
& =\int_{[0,1]^{j+1}} \int_{0}^{1} f\left(s_{1}-u_{1}, \ldots, s_{j}-u_{j} ;\left[s_{j+1}-u_{j+1}\right]-t_{j+1} ; J\right) d t_{j+1} d U
\end{aligned}
$$

by Fubini's theorem, and this becomes

$$
\begin{aligned}
& \int_{[0,1]^{j+1}}\left(\int_{0}^{1}\left(t_{j+1} \cdot f\right) d t_{j+1}\right)\left(s_{1}-u_{1}, \ldots, s_{j}-u_{j} ;\left[s_{j+1}-u_{j+1}\right] ; J\right) d U \\
& \quad\left(\text { note that if } n=1 \text { and } j=0, \int_{0}^{1}\left(t_{j+1} \cdot f\right) d t_{j+1} \text { is just } \chi_{[0,1]} * f\right) \\
& =\int_{[0,1]^{j+1}}\left(\int_{0}^{1}\left(t_{j+1} \cdot f\right) d t_{j+1}\right)_{(j+1)}\left(s_{1}-u_{1}, \ldots, s_{j}-u_{j} ; s_{j+1}-u_{j+1} ; J\right) d U \\
& =\left(\chi_{[0,1]^{j+1}} *\left(\int_{0}^{1}\left(t_{j+1} \cdot f\right) d t_{j+1}\right)_{(j+1)}\right)\left(s_{1}, \ldots, s_{j+1} ; m_{1}, \ldots, m_{n-j-1}\right) ;
\end{aligned}
$$

thus,

$$
\begin{aligned}
\left(\hat{\nu}_{(j+1)}\right)^{(j+1)}(f) & =\nu\left(\chi_{[0,1]^{j+1}} *\left(\int_{0}^{1}\left(t_{j+1} \cdot f\right) d t_{j+1}\right)_{(j+1)}\right) \\
& =\hat{\nu}\left(\left(\int_{0}^{1}\left(t_{j+1} \cdot f\right) d t_{j+1}\right)_{(j+1)}\right) .
\end{aligned}
$$

Now,

$$
\begin{aligned}
& \left(\int_{0}^{1}\left(t_{j+1} \cdot f\right) d t_{j+1}\right)_{(j+1)}\left(s_{1}, \ldots, s_{j+1} ; m_{1}, \ldots, m_{n-j-1}\right) \\
& \quad=\int_{0}^{1}\left(t_{j+1} \cdot f\right)\left(s_{1}, \ldots, s_{j} ;\left[s_{j+1}\right] ; m_{1}, \ldots, m_{n-j-1}\right) d t_{j+1} \\
& \quad=\int_{0}^{1} f\left(s_{1}, \ldots, s_{j} ;\left[s_{j+1}\right]-t_{j+1} ; m_{1}, \ldots, m_{n-j-1}\right) d t_{j+1} .
\end{aligned}
$$


Let $S=\left(s_{1}, \ldots, s_{j}\right)$ and $\left[s_{j+1}\right]-t_{j+1}=u$. Then

$$
\begin{aligned}
& \int_{\left[s_{j+1}\right]}^{\left[s_{j+1}\right]-1} f(S ; u ; J)(-d u)=\int_{\left[s_{j+1}\right]-1}^{\left[s_{j+1}\right]} f(S ; u ; J) d u \\
& \quad=\int_{\left[s_{j+1}\right]-1}^{s_{j+1}-1} f(S ; u ; J) d u+\int_{s_{j+1}-1}^{s_{j+1}} f(S ; u ; J) d u+\int_{s_{j+1}}^{\left[s_{j+1}\right]} f(S ; u ; J) d u .
\end{aligned}
$$

Substitute $v_{j+1}=s_{j+1}-u$ in the second integral and get

$(* * *)$

$$
\int_{\left[s_{j+1}-1\right]}^{s_{j+1}-1} f(S ; u ; J) d u+\int_{0}^{1} f\left(S ; s_{j+1}-v_{j+1} ; J\right) d v_{j+1}-\int_{\left[s_{j+1}\right]}^{s_{j+1}} f(S ; u ; J) d u .
$$

Let $T_{f}\left(S ; s_{j+1} ; J\right)=\int_{\left[s_{j+1}\right]}^{s_{j+1}} f(S ; u ; J) d u . T_{f}$ is easily seen to belong to

$$
\mathbf{L}^{\infty}\left(\mathbf{R}^{j+1} \oplus \mathbf{Z}^{n-j-1}\right),
$$

and $(* * *)$ is

$$
\begin{gathered}
T_{f}\left(S ; s_{j+1}-1 ; J\right)+\left(\int_{0}^{1}\left(v_{j+1} \cdot f\right) d v_{j+1}\right)\left(S ; s_{j+1} ; J\right)+T_{f}\left(S ; s_{j+1} ; J\right) \\
=\left(\int_{0}^{1}\left(v_{j+1} \cdot f\right) d v_{j+1}+\left(1_{j+1} \cdot T_{f}\right)-T_{f}\right)\left(S ; s_{j+1} ; J\right),
\end{gathered}
$$

so

$$
\begin{aligned}
\hat{\nu}\left(\left(\int_{0}^{1}\left(t_{j+1} \cdot f\right) d t_{j+1}\right)_{(j+1)}\right) & =\hat{\nu}\left(\int_{0}^{1}\left(v_{j+1} \cdot f\right) d v_{j+1}+\left(1_{j+1} \cdot T_{f}\right)-T_{f}\right) \\
& =\hat{\nu}\left(\int_{0}^{1}\left(v_{j+1} \cdot f\right) d v_{j+1}\right)+\hat{\nu}\left(1_{j+1} \cdot T_{f}\right)-\hat{\nu}\left(T_{f}\right) .
\end{aligned}
$$

By left invariance of $\hat{\nu}$, the second and third terms cancel, and we get

$$
\begin{aligned}
\nu\left(\chi_{[0,1]^{j+1}} * \int_{0}^{1}\left(v_{j+1} \cdot f\right) d v_{j+1}\right) & =\nu\left(\int_{0}^{1}\left(v_{j+1} \cdot f\right) d v_{j+1}\right) \\
& =\int_{0}^{1} \nu\left(v_{j+1} \cdot f\right) d v_{j+1}=\nu(f),
\end{aligned}
$$

so $\hat{\nu}_{(j+1)}$ is the preimage of $\nu$. Thus, if $M$ is a $\operatorname{LIM}$ on $\operatorname{UCB}\left(\mathbf{R}^{n}\right)$ we have

$$
\begin{aligned}
M(f) & =\left(\hat{M}_{(n)}\right)^{(n)}(f)=\left(\left(\left(\hat{M}_{(n)}\right)_{(n-1)}\right)^{(n-1)}\right)^{(n)} \\
& =\left(\cdots\left(\left(\hat{M}_{(n)}\right)_{(n-1)}\right) \hat{\cdots} \hat{(1)}^{(1) \cdots(n)}(f)\right. \\
& =\int_{[0,1]^{n}}\left(\cdots\left(\left(M_{(n)}\right)_{(n-1)}\right) \cdots\right)_{(1)}\left(\left(\left(t_{1}, \ldots, t_{n}\right) \cdot f\right)_{\mathrm{r}}\right) d t_{1} \cdots d t_{n} \\
& =\left\{\left(\cdots\left(\left(\hat{M}_{(n)}\right)_{(n-1)}\right) \cdots\right)_{(1)}\right\}_{e}(f) ;
\end{aligned}
$$

thus, $\left(\cdots\left(\left(\hat{M}_{(n)} \hat{)}_{(n-1)}\right) \cdots\right)_{(1)}\right.$ is the preimage of $M$, so $m \rightarrow m_{e}$ is surjective. Q.E.D.

5. The general abelian Lie group case. Let $G$ be an abelian analytic (i.e., connected Lie) group. Then $G$ is the direct product $\mathbf{R}^{n} \times\left(S^{1}\right)^{m}$, where $S^{1}$ is the circle, i.e., the 
multiplicative group of complex numbers with modulus 1 with the topology inherited from $\mathbf{R}$ (Exercise XIII 2: (i) $\Rightarrow$ (ii) of [7] followed by Corollary 4.2 of [7]). If $D$ is a discrete subgroup of $G$ such that $D \backslash G$ has finite right-invariant volume, then $D \backslash G$ is compact, being a topological group and equal to $G / D$. $D$ is a finitely generated abelian group (by the corollary to Proposition 3.7 of [8]) and is thus isomorphic to some $\mathbf{Z}^{n} \times\left\langle\alpha_{1}, \ldots, \alpha_{m}\right\rangle$, where each $\alpha_{i}$ is a primitive root of unity. By applying the following lemma twice, we get a bijection between LIM's on $l^{\infty}(D)$ and LIM's on $\operatorname{UCB}(G)$.

LEMMA 5.1. Let $G$ be the direct product of two locally compact amenable topological groups $N$ and $C$, with $C$ compact. For $f \in \mathrm{UCB}_{1}(N \times C)$, define

$$
m_{e}(f)=\int_{C} m\left((c \cdot f)_{\mathrm{r}}\right) d c
$$

Then $m \rightarrow m_{e}$ is a bijection between LIM's on $\operatorname{UCB}_{1}(N)$ and $\operatorname{UCB}_{1}(N \times C)$.

Proof. $m_{e}$ is easily seen to be a mean on $N \times C$. If $(k, \delta) \in N \times C$, we have

$$
\begin{aligned}
m_{e}((k, \delta) \cdot f) & =\int_{C} m\left((c \cdot(k, \delta) \cdot f)_{\mathrm{r}}\right) d c=\int_{C} m\left(((k, c \delta) \cdot f)_{\mathrm{r}}\right) d c \\
& =\int_{C} m\left(((k, 1)(1, c \delta) \cdot f)_{\mathrm{r}}\right) d c=\int_{C} m\left((c \delta \cdot f)_{\mathrm{r}}\right) d c
\end{aligned}
$$

by left invariance of $m$. Let $c \delta=b$. Then $d c=d b$ and $\int_{C} m\left((b \cdot f)_{\mathrm{r}}\right) d b=m_{e}(f)$. Thus, $m_{e}$ is left-invariant.

Now, define $f_{e}(k, \delta)=f(k)$. If $f \in \mathrm{UCB}_{1}(N)$ it is easily seen that

$$
f_{e} \in \mathrm{UCB}_{l}(N \times C) \text {. }
$$

Let $m_{1}$ and $m_{2}$ be LIM's on $\operatorname{UCB}_{1}(N)$ such that $m_{1 e}(g)=m_{2 e}(g)$ for each $g \in G$. For $f \in \operatorname{UCB}_{1}(N)$ and $c \in C, n \in N$, we have $c \cdot f_{e}(n, 1)=f_{e}\left(n, c^{-1}\right)=f(n)$, so $\left(c \cdot f_{e}\right)_{\mathrm{r}}=f$. Thus,

$$
\begin{aligned}
m_{1}(f) & =\int_{C} m_{1}(f) d c=\int_{C} m_{1}\left(c \cdot f_{e}\right) d c=m_{1 e}\left(f_{e}\right)=m_{2 e}\left(f_{e}\right) \\
& =\int_{C} m_{2}\left(c \cdot f_{e}\right) d c=\int_{C} m_{2}(f) d c=m_{2}(f),
\end{aligned}
$$

so $m \rightarrow m_{e}$ is injective.

To show surjectivity, let $M$ be a LIM on $\operatorname{UCB}_{1}(G)$ and define, for $f \in \operatorname{UCB}_{1}(N)$, $M_{0}(f)=M\left(f_{e}\right)$. Since

$$
\begin{gathered}
(n \cdot f)_{e}(k, \delta)=n \cdot f(k)=f\left(n^{-1} k\right)=f_{e}\left(n^{-1} k, \delta\right)=n \cdot f_{e}(k, \delta), \\
M_{0}(n \cdot f)=M\left((n \cdot f)_{e}\right)=M\left(n \cdot f_{e}\right)=M\left(f_{e}\right)=M(f),
\end{gathered}
$$

so $M_{0}$ is a $\operatorname{LIM}$ on $\operatorname{UCB}_{1}(N)$. If $g \in \operatorname{UCB}_{1}(G)$, then

$$
\begin{aligned}
\left(\int_{C}(c \cdot g)_{\mathrm{r}} d c\right)_{e}(k, \delta) & =\left(\int_{C}(c \cdot g)_{\mathrm{r}} d c\right)(k, 1)=\int_{C} c \cdot g(k, 1) d c \\
& =\int_{C} g\left(k, c^{-1}\right) d c
\end{aligned}
$$


Let $c=\delta^{-1} b$. Then $d c=d b$, and the integral becomes

$$
\begin{aligned}
\int_{C} g\left(k, b^{-1} \delta\right) d c & =\int_{C} b \cdot g(k, \delta) d b=\left(\int_{C}(b \cdot g) d b\right)(k, \delta) \\
& =\left(\int_{C}(c \cdot g) d c\right)(k, \delta) .
\end{aligned}
$$

Hence,

$$
\begin{aligned}
M_{0 e}(g) & =\int_{C} M_{0}\left((c \cdot g)_{\mathrm{r}}\right) d c=M_{0}\left(\int_{C}(c \cdot g)_{\mathrm{r}} d c\right)=M\left(\left(\int_{C}(c \cdot g)_{\mathrm{r}} d c\right)_{e}\right) \\
& =M\left(\int_{C}(c \cdot g) d c\right)=\int_{C} M(c \cdot g) d c=M(g),
\end{aligned}
$$

so $M_{0}$ is the preimage of $M$, and $m \rightarrow m_{e}$ is thus surjective.

THEOREM 5.2. Let $G$ be an abelian analytic group and $D$ a discrete subgroup of $G$ such that $G / D$ is compact. Then there is a bijection between LIM's on $l^{\infty}(D)$ and $L I M$ 's on $\mathrm{UCB}(G)$.

Proof. By the remarks preceding Lemma $5.1, G \cong \mathbf{R}^{n} \times\left(S^{1}\right)^{m}$ and $D \cong \mathbf{Z}^{n} \times$ $\left\langle\alpha_{1}, \ldots, \alpha_{m}\right\rangle$. If $D \cap \mathbf{R}^{n}=\mathbf{Z}^{n}$ is $N$ in the preceding theorem, and $C=\left\langle\alpha_{1}, \ldots, \alpha_{m}\right\rangle$, then $\int_{C}(c \cdot f)_{\mathrm{r}} d c$ is $(1 /|C|) \sum_{i=1}^{j_{1} \cdot j_{m}}\left(c_{i} \cdot f\right)_{\mathrm{r}}$, where $j_{k}$ is the order of $\alpha_{k}$ and $c_{i}=\alpha_{1}^{i_{1}}$ $\cdots \alpha_{m}^{i_{m}}, 0 \leqslant i_{k} \leqslant j_{k}$. Lemma 5.1 gives a bijection between LIM's on $l^{\infty}(D)$ and LIM's on $l^{\infty}\left(\mathbf{Z}^{n}\right)$. Theorem 4.1 gives a bijection between LIM's on $\operatorname{UCB}\left(\mathbf{R}^{n}\right)$ and LIM's on $\operatorname{UCB}\left(\mathbf{R}^{n}\right)$. Lemma 5.1 again gives a bijection between LIM's on $\operatorname{UCB}\left(\mathbf{R}^{n}\right)$ and LIM's on $\operatorname{UCB}(G)$. Composing these bijections gives the desired bijection between LIM's on $l^{\infty}(D)$ and LIM's on $\operatorname{UCB}(G)$. Q.E.D.

COROLlARY 5.3. Let $G$ be an abelian Lie group having finitely many components, and let $D$ be a discrete subgroup of $G$ such that $G / D$ is compact. Then there is a bijection between LIM's on $l^{\infty}(D)$ and LIM's on $\mathrm{UCB}(G)$.

Proof. Let $G_{0}$ be the connected component of the identity in $G$. By 24.45 of [6], $G$ is the direct product $G_{0} \times G / G_{0}$. If $D$ is a discrete subgroup of $G$ such that $G / D$ is compact, then $G_{0} / D \cap G_{0}$ is compact, being isomorphic to the closed subgroup $G_{0} D / D$ of $G / D$. By the corollary to Proposition 3.7 of [8], $D \cap G_{0}$ is finitely generated. Since $D / D \cap G_{0}=G_{0} D / G_{0} \subset G / G_{0}, D / D \cap G_{0}$ is finite, hence finitely generated. This implies that $D$ is finitely generated, since if $\left\{a_{1}, \ldots, a_{j}\right\}$ generate $D \cap G_{0},\left\{b_{1}\left(D \cap G_{0}\right), \ldots, b_{k}\left(D \cap G_{0}\right)\right\}$ generate $D / D \cap G_{0}$, and $d \in D$,

$$
\begin{aligned}
d\left(D \cap G_{0}\right) & =b_{1}^{l_{1}} \cdots b_{k}^{l_{k}}\left(D \cap G_{0}\right) \Rightarrow d b_{1}^{-l_{1}} \cdots b_{k}^{-l_{k}} \in D \cap G_{0} \\
& \Rightarrow d b_{1}^{-l_{1}} \cdots b_{k}^{-l_{k}}=a_{1}^{m_{1}} \cdots a_{j}^{m_{j}} \Rightarrow d=a_{1}^{m_{1}} \cdots a_{j}^{m_{j}} b_{1}^{l_{1}} \cdots b_{k}^{l_{k}} \\
& \Rightarrow\left\{a_{1}, \ldots, a_{j}, b_{1}, \ldots, b_{k}\right\}
\end{aligned}
$$

generate $D \Rightarrow D$ is finitely generated. By the fundamental theorem of abelian groups, $D \cong \mathbf{Z}^{k} \oplus\left(\oplus_{i=1}^{n} \mathbf{Z}_{m_{i}}\right)$, where $\oplus_{i=1}^{n} \mathbf{Z}_{m_{i}}$ is a finite direct sum of integers $\bmod m_{i}$ for various integers $m_{i} . D \cap G_{0}$ is thus $\cong \mathbf{Z}^{k} \oplus\left(\oplus_{i=1}^{q} \mathbf{Z}_{m_{i}}\right)$, where $q \leqslant n$. The exponent on $\mathbf{Z}$ is the same for $D$ and $D \cap G_{0}$, as $D / D \cap G_{0}$ is finite. 
Lemma 5.1 gives a bijection between LIM's on $l^{\infty}(D)$ and LIM's on $l^{\infty}\left(D \cap G_{0}\right)$. Theorem 5.2 gives a bijection between LIM's on $l^{\infty}\left(D \cap G_{0}\right)$ and LIM's on $\operatorname{UCB}\left(G_{0}\right)$. Lemma 5.1 again gives a bijection between LIM's on $\operatorname{UCB}\left(G_{0}\right)$ and LIM's on $\mathrm{UCB}(G)$. Composing these bijections gives the desired result. Q.E.D.

By Theorem 5.1 of [1] there are at least $2^{c}$ LIM's on $\mathrm{CB}(G)$ for any locally compact, noncompact amenable group, where $c=$ card $\mathbf{R}=$ cardinality of $\mathbf{R}$. If $G$ is an abelian Lie group with finitely many components, we can be more precise about LIM's on $\operatorname{UCB}(G)$.

COROLlaRY 5.4. Let $G$ be a noncompact abelian Lie group having finitely many connected components. Then there are exactly $2^{c} L I M$ 's on $\mathrm{UCB}(G)$.

Proof. Let $D$ be a discrete subgroup of $G$ such that $G / D$ is compact. (e.g., since $G \cong \mathbf{R}^{n} \times\left(S^{1}\right)^{m} \times G / G_{0}$, we can let $\left.D=\mathbf{Z}^{n}\right)$. By Theorem 1 of [2], the cardinality of the LIM's on $l^{\infty}(D)$ is $2^{c}$, since $c=2^{\text {card } D}$. The conclusion thus follows from Corollary 5.3. Q.E.D.

6. Remarks. We are unable to generalize the proof of Theorem 4.1 to the general Lie group case or even to the solvable case. It is conceivable that the proof could be extended to at least the simply connected solvable case, using the fact that such a group is (isomorphic to) semidirect products of $\mathbf{R}$. The difficulty lies in showing the means constructed are invariant at each stage.

More generally, if $G$ is analytic and has a faithful, finite-dimensional, continuous representation, we know $G$ is isomorphic to a semidirect product $N \times_{\eta} H$, where $N$ is simply connected and solvable and $H$ is reductive. If $G$ is also solvable so is $H$, and by Theorem XVIII 4.4 of [7], $H / Z(H)$ is semisimple, where $Z(H)$ is the center of $H$, but $H / Z(H)$ being solvable also implies it is trivial, so $Z(H)=H$. Also, by the same theorem, $H$ is compact, and being abelian implies $H \cong\left(S^{1}\right)^{m}$. It would seem that if one could prove that $m \rightarrow m_{e}$ is bijective for $G$ simply connected and solvable, a generalization of Theorem 5.1 would produce a bijection between LIM's on $l^{\infty}(D)$ and on $\operatorname{UCB}_{1}(G)$. The following example illustrates the problem.

EXAMPLE 6.1. Let $G$ be the semidirect product $\mathbf{R}^{2} \times{ }_{\eta} S^{1}$, where

$$
\eta\left(e^{i \theta}\right)(x, y)=(x \cos \theta+y \sin \theta,-x \sin \theta+y \cos \theta)
$$

(i.e., $\eta\left(e^{i \theta}\right)$ rotates $(x, y)$ clockwise through an angle of $\theta$ radians). Let $M$ be a LIM on $\operatorname{UCB}_{1}(G)$. We would like a $\operatorname{LIM} \mu$ on $\operatorname{UCB}\left(\mathbf{R}^{2}\right)$ such that $\mu_{e}(g)=M(g)$ for every $g \in \operatorname{UCB}_{1}(G)$. If $f \in \operatorname{UCB}\left(\mathbf{R}^{2}\right)$, we have

$$
f(r)=\int_{S^{1}} f(r) d s=\int_{S^{1}} s \cdot f_{e}(r) d s
$$

and

$$
\begin{aligned}
M\left(f_{e}\right) & =\mu_{e}\left(f_{e}\right)=\int_{S^{1}} \mu\left(\left(s \cdot f_{e}\right)_{\mathrm{r}}\right) d s \\
& =\mu\left(\int_{S^{1}}\left(s \cdot f_{e}\right)_{\mathrm{r}} d s\right)=\mu(f) .
\end{aligned}
$$


Thus, $\mu(f)$ must be defined to be $M\left(f_{e}\right)$, but we need to show $\mu$ is $\mathbf{R}^{2}$-left-invariant. Given $\left(r_{1}, r_{2}\right) \in \mathbf{R}^{2}$, we would like to have $\left(\left(r_{1}, r_{2}\right) \cdot f\right)_{e}=\left(t_{1}, t_{2} ; e^{i \phi}\right) \cdot f_{e}$ for some $t_{1}, t_{2}, \phi$ so that

$$
\mu\left(\left(r_{1}, r_{2}\right) \cdot f\right)=M\left(\left(\left(r_{1}, r_{2}\right) \cdot f\right)_{e}\right)=M\left(\left(t_{1}, t_{2} ; e^{i \theta}\right) \cdot f_{e}\right)=M\left(f_{e}\right)=\mu(f) ;
$$

however, this is impossible for even $r_{1}=\pi, r_{2}=0$. Simple calculations show

$$
\left(t_{1}, t_{2} ; e^{i \phi}\right) \cdot f_{e}\left(x_{1}, x_{2} ; e^{i \theta}\right)=\left(t_{1}, t_{2} ; 1\right) \cdot f_{e}\left(x_{1}, x_{2} ; e^{i \theta}\right)
$$

for all $\phi$.

Now, let $f\left(x_{1}, x_{2}\right)=\sin \left(x_{1}+x_{2}\right)$. Clearly $f \in \operatorname{UCB}\left(\mathbf{R}^{2}\right)$, and simple calculations show

$$
\begin{aligned}
& \left(t_{1}, t_{2} ; 1\right) \cdot f_{e}\left(x_{1}, x_{2} ; e^{i \theta}\right) \\
& \quad=\sin \left(\left(x_{1}-t_{1}\right)(\cos \theta+\sin \theta)-\left(x_{2}-t_{2}\right)(\sin \theta-\cos \theta)\right)
\end{aligned}
$$

and

$$
\begin{aligned}
& \left(\left(s_{1}, s_{2}\right) \cdot f\right)_{e}\left(x_{1}, x_{2} ; e^{i \theta}\right) \\
& \quad=\sin \left(x_{1}(\cos \theta+\sin \theta)-x_{2}(\sin \theta-\cos \theta)-\left(s_{1}+s_{2}\right)\right) .
\end{aligned}
$$

Let $s_{1}=\pi, s_{2}=0$, and $\theta=\pi$. Equating $(*)$ and $(* *)$ implies that

$$
t_{1}+t_{2}=2 n \pi-\pi \text {. }
$$

If $\theta=\pi / 2$, we get

$$
t_{1}-t_{2}=2 m \pi+\pi \text {. }
$$

Solving for $t_{1}$ in ( $\dagger$ ) and (††) we get $2 t_{1}=2 \pi(n+m)$, or $t_{1}=\pi(n+m)$, so $t_{2}=2 n \pi-\pi-\pi(n+m)=\pi(n-m)-\pi$; if $\theta=\pi / 4$, equate $(*)$ and $(* *)$ and get

$$
\begin{aligned}
\sin \left(\sqrt{2}\left(x_{1}-t_{1}\right)\right) & =\sin \left(\sqrt{2} x_{1}-\pi\right) \Rightarrow \sqrt{2}\left(x_{1}-t_{1}\right)+2 k \pi=\sqrt{2} x_{1}-\pi \\
& \Rightarrow \sqrt{2} t_{1}=\pi+2 k \pi \Rightarrow \sqrt{2}(n+m)-2 k=1,
\end{aligned}
$$

impossible for $n, m, k \in \mathbf{Z}$. Thus, $((\pi, 0) \cdot f)_{e} \neq\left(t_{1}, t_{2} ; e^{i \phi}\right) \cdot f_{e}$. This does not make surjectivity impossible, since for $\mu$ to be $\mathbf{R}^{2}$ invariant for all $M$ we need $M\left(f_{e}-\left(\left(s_{1}, s_{2}\right) \cdot f\right)_{e}\right)=0$; i.e., $f_{e}-\left(\left(s_{1}, s_{2}\right) \cdot f\right)_{e}$ is a uniform limit of functions of the form $\sum c_{i}\left(g_{i}-x_{i} \cdot g_{i}\right)$ for $x_{i} \in G, c_{i} \in \mathbf{R}, g_{i} \in \mathrm{UCB}_{1}(G)$ by Theorem 2.3.

If $G$ is any analytic group, $G \cong\left(\operatorname{Rad} G \times{ }_{\theta} S\right) / \Delta$, where $\Delta$ is discrete and central, $\operatorname{Rad} G=\operatorname{radical}$ of $G$, and $S$ is a maximal semisimple analytic subgroup of $G$. If $G$ is amenable, so is $S$ and, being semisimple, is thus compact. If follows that $\Delta$ must be finite. If $\operatorname{Rad} G \cong N \times_{\eta}\left(S^{1}\right)^{m}$, it seems reasonable that an appropriate generalization of Lemma 5.1 applied twice would yield bijections between LIM's on $\operatorname{UCB}_{1}(N)$, $\mathrm{UCB}_{1}(\operatorname{Rad} G)$, and $\mathrm{UCB}_{1}(G)$. Thus

CONJECTURE. If $G$ is an analytic group having a faithful, finite-dimensional, continuous representation, and $D$ is a discrete subgroup of $G$ such that $D \backslash G$ has right-invariant finite volume, there is a bijection between LIM's on $l^{\infty}(D)$ and LIM's on $\mathrm{UCB}_{1}(G)$. 


\section{BIBLIOGRAPHY}

1. C. Chou, On topologically invariant means on a locally compact group, Trans. Amer. Math. Soc. 151 (1970), 443-456.

2. The exact cardinality of the set of invariant means on a group, Proc. Amer. Math. Soc. 55 (1976), 103-106.

3. M. M. Day, Amenable semigroups, Illinois J. Math. 1 (1957), 509-544.

4. N. Dunford and J. T. Schwartz, Linear operators, Interscience, New York, 1967.

5. F. P. Greenleaf, Invariant means on topological groups, Van Nostrand, Princeton, N. J., 1969.

6. E. Hewitt and K. A. Ross, Abstract harmonic analysis, vol. 1, Springer-Verlag, Berlin and New York, 1979.

7. G. Hochschild, The structure of Lie groups, Holden-Day, San Francisco, Calif., 1965.

8. M. S. Raghunathan, Discrete subgroups of Lie groups, Springer-Verlag, Berlin and New York, 1972.

Department of Mathematics, University of Virginia, Charlottesville, Virginia 22903

Current address: Department of Mathematics, Randolph-Macon Woman's College, Lynchburg, Virginia 24503 